\title{
Importancia de las competencias digitales directivas para la formación de talentos en la Industria 4.0
}

\section{Importance of directive digital competences for talent training in Industry 4.0}

http://dx.doi.org/10.17981/cultedusoc.13.1.2022.11

Recibido: 17 de noviembre de 2020. Aceptado: 8 de febrero de 2021. Publicado: 24 de enero de 2022.

Jorge Carlos Canto-Esquivel

Tecnológico Nacional de México. Mérida (México) jorge.ce@merida.tecnm.mx

Jennifer Mul-Encalada

Universidad Autónoma de Yucatán. Mérida (México) jeni.mul@correo.uady.mx

\section{Ruth Noemí Ojeda-López}

Universidad Autónoma de Yucatán. Mérida (México) ruth.ojeda@correo.uady.mx

Para citar este artículo:

Canto-Esquivel, J., Mul-Encalada, J. y Ojeda-López (2022). Importancia de las competencias digitales directivas para la formación de talentos en la Industria 4.0. Cultura, Educación y Sociedad, 13(1), 177-192. DOI: http://dx.doi.org/10.17981/cultedusoc.13.1.2022.11

\section{Resumen}

Introducción: La participación de los docentes en la formación de los nuevos talentos para la industria 4.0 requiere de visiones y acciones disruptivas para lograr la transformación digital de las empresas donde laborarán sus estudiantes. El presente estudio analiza la importancia que asignan los docentes a las competencias digitales requeridas para dirigir las empresas dentro de la cuarta revolución industrial. Metodología: Estudio cuantitativo de tipo descriptiva por medio de la técnica de la encuesta a través de un cuestionario en línea a 152 docentes universitarios del estado de Yucatán (México). Resultados y discusión: Se evidencia que los docentes valoran con mayor importancia las competencias digitales directivas relacionadas con la innovación, nuevos productos y los clientes; seguidas de la comprensión y gestión de la economía global digital; las tecnologías de la información y comunicación; la organización de las empresas digitales; la gestión, aplicación y financiación de nuevos modelos de negocios basados en la economía digital y los activos intangibles. Conclusiones: La participación de los docentes es primordial en la formación de competencias que requerirán las empresas en el proceso de cambio.

Palabras clave: Competencias digitales; docencia; educación y tecnología; sociedad futura; enseñanza superior

\section{Abstract}

Introduction: The participation of teachers in the training of new talents for Industry 4.0 requires visions and disruptive actions to achieve the digital transformation of the companies where their students will work.This study analyzes the importance that teachers assign to the digital skills required to run companies within the fourth industrial revolution. Methodology: Quantitative descriptive study using the survey technique through an online questionnaire to 152 university teachers from the state of Yucatán (Mexico). Results and discussion: It is evidenced that teachers value with greater importance the managerial digital competences related to innovation, new products and customers; followed by understanding and managing the global digital economy; information and communication technologies; the organization of digital companies; the management, application and financing of new business models based on the digital economy and intangible assets. Conclusions: The participation of teachers is essential in the formation of competencies that companies will require in the process of change.

Keywords: Digital skills; teaching; education and technology; future society; university education 


\section{INTRODUCCIÓN}

La cuarta revolución industrial generará varias actividades transversales dentro de los empleos, los cuales requerirán actitudes, habilidades y conocimientos que no son precisamente de disciplinas únicas. Estos distintos roles requerirán nuevas habilidades, tanto sociales como técnicas. La formación de competencias necesitará ser reorientada en los sistemas educativos para generar las capacidades que requerirán los egresados para ser contratados. La dicotomía de las ciencias y las humanidades, las certificaciones técnicas y la formación universitaria, implicarán retos en la educación con los modelos actuales.

En México, la Secretaría de Economía (SEECO, 2016) manifestó contar con la infraestructura adecuada para el inicio de la industria 4.0, observando las siguientes fortalezas: Amplia oferta académica para generar talento especializado, creciente economía de servicios, industrias de alta tecnología para generación de demanda y disponibilidad de recursos humanos creativos y una industria electrónica que se ha posicionado como exportador líder altamente sofisticado entre los países latinoamericanos.

Los cambios digitales en las sociedades de los países desarrollados se vendrán dando en un breve lapso. Las estrategias para alcanzar la transformación digital de las sociedades ya están establecidas por los países desarrollados que se encuentran preparando a su capital humano para las modificaciones que se pretenden para este futuro cercano. Como Bauman (2003) establece, el problema es que el futuro es tan difícil de diagnosticar que el tomador de decisiones se siente impotente en el proceso productivo y debe estar preparado con las competencias necesarias para la respuesta al sistema.

Uno de los principales datos a considerar en esta revolución tecnológica es la cantidad de usuarios de la internet. México cuenta con 6 millones 448 mil usuarios conectados a la red. Para el 2015 aproximadamente 77 millones 711 mil mexicanos ya eran usuarios de telefonía celular y aproximadamente 44 millones 479 mil se conectaban a través de un teléfono inteligente (Instituto Nacional de Estadísticas, Geografía e Información-INEGI, 2017).

La globalización puede diferenciar diversos niveles de significados, dimensionándolos en lo político, lo ideológico-cultural, lo económico y lo técnico. Por una parte, en lo político, la globalización ha transformado el orden mundial debido al constante peligro del liderazgo de las naciones que sustentaban la supremacía del capitalismo en pleno auge de la crisis sistémica, civilizacional y financiera. La soberanía mundial al fragmentarse ha generado nuevas luchas, resistencias y conflictos al interior de los intereses de cada nación que aspira al control político, económico, financiero y cultural del nuevo sistema mundial (Velásquez, 2020).

En vista de que la globalización es un proceso objetivo de estructuración del conjunto de la economía, sociedades, instituciones y culturas; se plantea la idea de que requiere también de una cultura global, la homogenización cultural del mundo. Este aspecto genera críticas durante el proceso, por un lado, la superación de los atavismos históricos identitarios, para fundirnos en una especie de cultura universal indiferenciada, ligada a la especie humana; y por otro lado la imposición de una homogeneización cultural (Castells, 2010). 
En lo económico se refiere al proceso de liberación de bienes, servicios y capitales, así como a la internacionalización de la producción. En lo técnico se refiere a la implantación de las nuevas tecnologías que han sido vinculadas principalmente en relación con la transferencia de información (Hirsch, 1996). Las habilidades para aprovechar las tecnologías para beneficios económicos son necesarias en esta era.

De acuerdo con el contexto y desarrollo de un país, las competencias directivas que proporcionen a las empresas capacidades de subsistir y competir, serán adecuadas. Estas competencias relacionadas con la innovación, las tecnologías de la información, los modelos de negocio y las formas de organizar el trabajo varían de acuerdo con el nivel de digitalización de las empresas y el desarrollo de la industria (Lombardero, 2015). Incluso la falta de competencias adecuadas pudiera hacer desaparecer las empresas locales, evitando el desarrollo.

Las empresas demandan personas con perfiles profesionales concretos y especializados los cuales puedan adaptarse a los cambios que el sector va generando. La velocidad de adaptación del mundo educativo es diferente de la del productivo debido a la rigidez curricular establecida por las instancias reguladoras, la legislación académica, y a las dificultades de formación continua y participación del profesorado en empresas a través de estancias (López y Escudero, 2016).

Los cambios en las competencias relacionadas con el empleo requerirán de procesos de enseñanza y aprendizaje distintos e incluso disruptivos. En ese sentido, la educación media superior y superior presentan hoy en sus planes de estudio competencias digitales básicas para el desempeño de sus estudiantes en relación con estos cambios digitales.

Por lo anterior, el objetivo de este estudio es analizar la importancia que otorgan los docentes a las competencias digitales que requieren los estudiantes como futuros talentos laborales para dirigir las empresas del estado de Yucatán dentro de la cuarta revolución industrial.

\section{REVISIÓN DE LA LITERATURA}

De acuerdo con Naji (2018), las competencias digitales son todas aquellas habilidades, capacidades y aptitudes relacionadas con la aplicación, el uso eficaz, útil, práctico y seguro de nuevas tecnologías, tanto en el entorno profesional, social, formativo, familiar, ocio, etcétera. Una competencia implica diferentes grados de complejidad los cuales exigen conocimientos procedimentales y condicionales, y a su vez, las capacidades están constituidas por habilidades necesarias para una ejecución compleja. Estas competencias, basadas actualmente en las Tecnologías de la información y de la Computación-TIC, se adquieren a través de múltiples instituciones como la familia, la escuela y los medios de comunicación, permitiendo que los sujetos no sólo accedan a las tecnologías, sino que se desempeñen con ella de manera creativa y crítica en la producción de contenidos para las diversas plataformas de conocimiento (Vera-Noriega, Rodríguez-Carvajal, y Martínez-García, 2017).

Según la prospectiva internacional del World Economic Forum-WEF (2016), las áreas laborales relacionadas con la computación y las matemáticas tendrían un crecimiento más acelerado en el periodo del 2015-2020, seguidos por la arquitectura y la ingeniería. Este crecimiento se esperaba debido al desarrollo tecnológico que tiene que ver directamente con 
la industria 4.0. Los principales cambios que se presentarán en el ámbito socioeconómico del mundo serán en la naturaleza del trabajo y la flexibilidad de este; siendo los servicios profesionales, la industria básica, de infraestructura y la energía, las actividades con más transformaciones (WEF, 2016).

Estas predicciones se estimaban para que se presenten en la generalidad de las industrias a más tardar en el año 2020 (Vera-Noriega et al., 2017). Los hechos recientes derivados de la crisis sanitaria del COVID-19 en este 2020 han afectado las tendencias vigentes de los mercados, muchas de ellas, si bien no todas, relacionadas con la digitalización. Durante el tiempo de confinamiento se ha transformado la forma de trabajar, sobre todo la aplicación del teletrabajo y la generación de nuevos empleos en actividades básicas, algunas de ellas facilitadas por plataformas digitales (Weller, 2020). La aceleración de estas adopciones tecnológicas debido al confinamiento, según este mismo autor, también derivó en la destrucción de empleos y desigualdad, pues hay mayores riesgos de perder el empleo para personas con menores niveles de educación, es decir entre personas con diferentes niveles de habilidades digitales.

De esta manera los empleos que tendrían un crecimiento negativo acelerado, según el WEF (2016), son los relacionados con las tareas administrativas y de oficina, y la operación manual en fábricas y manufactura, los cuales se esperaba que fueran disminuyendo debido al alto número de actividades repetitivas que se llevan a cabo en su desarrollo. Es decir, las competencias laborales que se desarrollen en el futuro, necesitarán de niveles cognoscitivos más elevados y que no sean fácilmente remplazados por la inteligencia artificial o la automatización.

Según Franco (2015), la Industria 4.0 se basa en tres aspectos fundamentales: Individualización, descentralización y creación de redes. La individualización se refiere a la satisfacción de un mercado heterogéneo donde se requiere alcanzar una economía de escala y considerar el ciclo de vida del producto, se conoce también como "personalización masiva" (mass customization). La personalización masiva es una estrategia de producción que se centra en los procesos flexibles. De este mismo modo, la descentralización productiva consiste en la organización de la producción de manera tal que, determinadas partes del proceso u operaciones se encarguen a terceros, esto permite hacer frente a un entorno de cambios complejos y permite la adaptación a la producción de productos personalizados. Por lo que la creación de redes facilitará la interacción entre empresas, universidades y administraciones públicas de cara a la implantación de modelos de manufactura avanzada, permitiendo la cooperación y colaboración entre los diferentes actores, así como el intercambio de información.

Por su parte, Castillo (2016) establece que los ingredientes esenciales de la industria 4.0 serán los siguientes: Máquinas trabajando con humanos de manera colaborativa; fabricación adaptable a las necesidades específicas de los clientes; instalaciones autónomas, plantas auto gestionables que se puedan configurar de manera flexible para dar respuesta a situaciones de producción cambiantes según la demanda del mercado; información en tiempo real, que permita que todas las áreas de la empresa se integren para mejorar la eficiencia y controlar a tiempo posibles errores y fallos; y por último, la fusión entre lo real y lo virtual, proceso que volverá a la fábrica inteligente al tener una alta capacidad de adaptación, y eficiencia en el uso de sus recursos simulando escenarios y observando de manera virtual las posibilidades. 
Por otro lado, la comunicación digital permite potenciar la cercanía de los públicos, las herramientas digitales de comunicación ofrecen alternativas para respaldar los procesos comerciales y productivos. Sin embargo, estas herramientas se fundamentan en la interacción y retroalimentación constante para establecer un diálogo y construir relaciones alrededor de la organización o la marca (Pineda, 2020). Las nuevas organizaciones digitales requieren de talentos que puedan contribuir con las soluciones a las demandas de colaboración y construcción conjunta de contenido.

La digitalización de las empresas supone un cambio radical en la forma de trabajo actual. Los modelos de negocio, la relación con los clientes, el valor de los datos, intra emprendimiento, las redes de valor, la agilidad y la experimentación son palancas que caracterizan a la industria 4.0 (López y Escudero, 2016). En este sentido, Naji (2018) expresa que, de las competencias requeridas para el futuro, las digitales muy probablemente se desarrollen a través de la combinación del aprendizaje formal e informal, es decir con la práctica en el trabajo y en el tiempo libre.

Según el International Telecommunication Union (ITU, 2018) estas habilidades digitales avanzadas son adquiridas normalmente en la educación convencional avanzada. Se suman a estas habilidades las del emprendimiento digital. Del mismo modo indica que las empresas digitales se caracterizan por su alta intensidad de utilización de las nuevas tecnologías digitales para mejorar el funcionamiento empresarial, inventar nuevos modelos de negocio, agudizar la inteligencia empresarial e interacciones con clientes e interesados. Los cambios de las empresas y de las actividades económicas están dándose a una velocidad que las universidades no pueden alcanzar o que en sus procesos de planeación no se consideraron con anterioridad.

Es por esta razón que desarrollar habilidades como aprender a aprender se vuelve fundamental en este cambio de era laboral. El aprendizaje permanente desarrolla de manera continua conocimiento y habilidades a lo largo de la vida, principalmente después de la educación formal; éste se relaciona directamente con la autodirección del aprendizaje y el aprendizaje autónomo. Para este desarrollo auto dirigido se presentan cuatro ámbitos a considerar: la autonomía personal, la voluntad y la capacidad para gestionar los esfuerzos de aprendizaje, la búsqueda independiente de aprendizaje sin apoyo institucional, y el control de la instrucción por parte del aprendiz. Por eso la necesidad de establecer el foco en entornos de aprendizaje diversos, descartando la idea de que el aprendizaje sólo se promueve de manera formal, aceptando y fomentando el aprendizaje abierto y en línea (Carrizosa, 2018).

Por otro lado, las universidades se encuentran en la búsqueda constante de una vinculación con el sector productivo y el gobierno, para el intercambio de necesidades de perfiles profesionales. En la actualidad las instituciones educativas juegan un papel fundamental en el proceso de integración tecnológica, es en las aulas donde los empleados calificados adquieren las competencias digitales mínimas necesarias para poder desempeñarse en sus labores cotidianas (Carruyo, Ureña y Quiñones, 2017).

En el ámbito educativo el uso pedagógico de TIC para la adquisición de competencias por parte de los alumnos depende de las TIC utilizadas por el profesor (Herrero, 2014). En la medida en la que los profesores utilicen nuevas herramientas digitales, los estudiantes también lo harán (Zempoalteca, Barragán, González y Guzmán, 2017). Muchas herramientas son usadas por el estudiantado por demanda del profesorado. 
Es así como, las universidades con centros de investigación y desarrollo tecnológico, también son centros de difusión de conocimiento y formación de profesionales (Quintero, 2017). La transformación que hoy se necesita, además de la técnica-instrumental, necesita prestar especial atención en la formación constante de especialistas para la generación, la administración y la extensión del conocimiento (Cobo, 2010). No hay duda que, las universidades son pilares en la formación técnica de los futuros profesionales, sin embargo, los cambios en el ámbito laboral están exigiendo una formación para la adaptación profesional, la re-educación y la educación continua.

Además, la innovación se ha convertido en un proceso fundamental en las universidades. El verdadero desafío es el de potenciar la capacidad de resolver problemas reales, generando el conocimiento necesario y el espíritu crítico que aporte soluciones al mundo (López, 2019). Para las empresas los nuevos clientes y usuarios no sólo son compradores, sino que se convierten en fuente y generadores de ideas para la innovación, e incluso son participantes en diferentes etapas de los proyectos empresariales (Herrera e Hidalgo, 2019). La innovación se convierte entonces en un intercambio de conocimiento entre los actores académicos y los del sistema económico como los empresarios, los clientes y los usuarios.

Area (2010) indica que, para salir victoriosos de los retos digitales, las universidades pueden tomar como razones y argumentos para la enseñanza de las competencias digitales para acceder a la información, las siguientes:

1. El crecimiento exponencial de la producción de conocimiento en las áreas del saber, los ámbitos científico, técnico, humanístico, artístico o social se encuentran entrelazándose entre disciplinas.

2. Los repositorios digitales de información, como bibliotecas digitales, bases de datos, portales digitales, publicaciones digitales, blogs y redes sociales se han dispuesto para el fácil acceso de las personas que sepan utilizar estas herramientas.

Para el autor las teorías pedagógicas y del aprendizaje del conocimiento deben ser construidas por el propio individuo desde la vivencia de experiencias, con la interacción de otros sujetos y dirigido a la acción. Por esta razón y gracias a las tecnologías de la información en los procesos de traducción el idioma ha dejado de ser un elemento limitador de la expresión de ideas y la comunicación efectiva de éstas. Los medios digitales han permitido que los sentimientos, las opiniones y los conocimientos vayan adoptando formas y lenguajes múltiples que se proyectan en textos escritos, medios audiovisuales o archivos multimedia; que permiten a los estudiantes participar en esta sociedad de conocimiento de manera autónoma sin importar la disciplina que se esté estudiando.

De este modo, el uso de herramientas virtuales para la enseñanza universitaria a través de plataformas educativas de e-learning, docencia virtual, educación semipresencial o b-learning, MOOC (Massive Online Open Courses) entre otros recursos permite a los estudiantes universitarios complementar su proceso de enseñanza aprendizaje. Al cual pueden acceder gracias al dominio de las competencias digitales que necesitarán a lo largo de su vida profesional. 


\section{Metodología}

El objetivo propuesto en este trabajo es analizar la importancia que otorgan los docentes a las competencias digitales que requieren los futuros talentos egresados para dirigir las empresas dentro de la cuarta revolución industrial. Con esta finalidad se plantea una investigación cuantitativa, descriptiva, de corte transversal y no experimental (Bernal, 2010). Los resultados proyectan la configuración de estrategias docentes adecuadas para dirigir los esfuerzos académicos que preparen a los futuros talentos empresariales.

Las variables son las competencias digitales directivas discriminadas así:

a. Competencias relacionadas con las características de la economía digital y los modelos de negocio.

b. Competencias sobre las TIC.

c. Competencias relacionadas con la innovación, los nuevos productos y la relación con el cliente.

d. Competencias relacionadas con las estructuras organizativas de las empresas digitales.

El instrumento diseñado por Lombardero (2015) y que evalúa la importancia de las competencias digitales directivas centrándose en las cuatro variables establecidas incluye cinco apartados donde el primero se centra en los datos demográficos y académicos de los docentes. El apartado inicial son preguntas de opción múltiple; en los siguientes las variables se han graduado utilizando escalas tipo Likert de siete puntos codificados de 1 al 7 , donde 1 equivale a poco importante y 7 a muy importante. El Instrumento consta de un total de 40 competencias.

Se incluye en el estudio a docentes que imparten clases en Instituciones de Educación Superior-IES con una matrícula mayor a mil estudiantes en licenciaturas relacionadas con las áreas de finanzas, administración e ingeniería. Se determinó de acuerdo con el registro de IES de la Secretaria de Educación del Estado de Yucatán-SEGEY, que para el ciclo 2017-2018 existían 12 institutos tecnológicos y universidades tecnológicas, y 26 instituciones de educación superior incorporadas a la SEGEY que cumplían con el criterio de inclusión al estudio, en las cuales se encuestaron a sus docentes.

El instrumento diseñado por Lombardero (2015) evalúa la importancia de las competencias digitales directivas centrándose en cuatro dimensiones relacionadas con la industria 4.0. Estas dimensiones son: 1) modelos de negocio y economía digital, donde se abordan las competencias relacionadas con el entendimiento de estos temas; 2) tecnologías, se refiere al conocimiento de las TIC y a los pilares tecnológicos de la industria 4.0; 3) innovación, son las competencias que se requieren para el proceso de innovación de nuevos productos y clientes; y 4) organizativas, las cuales hacen referencia a la gestión de los cambios organizacionales debido al uso de las tecnologías.

Se determina la confiabilidad del instrumento a través del alfa de Cronbach, obteniendo resultados favorables de $.950, .935, .940$ y .937 ; respectivamente para cada grupo de competencias. Se realiza la invitación a todos los directivos de estas instituciones para que participaran sus docentes, logrando una muestra no probabilística por conveniencia de 152 docentes. La recolección de datos se aborda vía encuestas en línea. La codificación y procesamiento de la información en el programa Statistical Package for Social Sciences (SPSS, versión 27). 


\section{Resultados}

Se obtiene información de 152 docentes del estado de Yucatán, de los cuales el 29.6\% eran mujeres y el 70.4\% hombres. Participaron docentes principalmente del Instituto Tecnológico de Mérida con un 50\% de la muestra, 12.5\% pertenecientes a la Universidad Modelo, 7.9\% al Instituto Tecnológico Superior de Motul, 4.6\% a la Universidad Autónoma de Yucatán y el 25\% restante de otras universidades. El nivel máximo de estudio que presentan los docentes se distribuye de la siguiente manera: $2.0 \%$ cuenta con postdoctorado, $19.7 \%$ con doctorado, $46.1 \%$ con maestría, $19.7 \%$ con licenciatura, $8.6 \%$ es técnico superior universitario y el 3.9\% técnico. La mayoría de los docentes encuestados se declararon en una relación laboral de empleado, el 73.1\% de ellos, el 10.5\% declaró trabajar por cuenta propia y el $16.4 \%$ declaró hacerlo por cuenta propia y como empleado.

Con respecto a la categoría profesional, dentro de la institución educativa, el $57.9 \%$ es docente, el $15.1 \%$ es mando medio, el $13.2 \%$ es investigador, el $7.2 \%$ técnico y el $6.6 \%$ restante es directivo o ejecutivo. Solo el 6.6\% de las IES donde laboran los docentes de la muestra tienen más de 1000 trabajadores, $69.7 \%$ entre 250 y 999 trabajadores, el 15.1\% entre 100-249 trabajadores y 8.6\% tiene menos de 99 trabajadores. Al identificar su organización, los docentes declaran en el $68.4 \%$ de las veces que es una organización con actividades tradicionales y digitales, el $22.4 \%$ que es de nuevas tecnologías, el $5.9 \%$ de la economía tradicional y solo el 3.3\% de la economía digital.

En la Tabla 1 se presentan los valores de las medias para cada dimensión obtenida ordenados alfabéticamente. Como se puede observar las competencias de mayor a menor importancia para los docentes fueron:

1. Las relacionadas con la innovación, los nuevos productos y los clientes (DC1).

2. Las relacionadas con comprensión y gestión de la economía global digital (DA2).

3. Las relacionadas con las TIC (DB1).

4. Las relacionadas con la organización de las empresas digitales (DD1).

5. Las relacionadas con la gestión, aplicación y financiación de nuevos modelos de negocios basados en la economía digital y los activos intangibles (DA1).

TABLA 1.

Informe de medias para la muestra de docentes.

\begin{tabular}{llllll}
\hline & \multicolumn{1}{c}{ DA1 } & \multicolumn{1}{c}{ DA2 } & \multicolumn{1}{c}{ DB1 } & \multicolumn{1}{c}{ DC1 } & \multicolumn{1}{c}{ DD1 } \\
\hline Media & 5.6671 & 5.9961 & 5.8454 & 6.0278 & 5.8048 \\
$\mathrm{~N}$ & 152 & 152 & 152 & 152 & 152 \\
Desv. & 1.07788 & .97477 & 1.27343 & .99045 & 1.01618 \\
Mínimo & 1.20 & 1.60 & 1.00 & 1.33 & 1.22 \\
Máximo & 7.00 & 7.00 & 7.00 & 7.00 & 7.00 \\
\hline
\end{tabular}

Fuente: Elaboración propia basada en los datos obtenidos del estudio. 
Se realiza la prueba $t$ de muestras emparejadas para determinar las diferencias de medias entre las competencias. Las estadísticas descriptivas de los pares de competencias ordenadas de mayor a menor se muestran en la Tabla 2.

TABLA 2.

Estadisticas de muestras emparejadas para docentes.

\begin{tabular}{llllcl}
\hline & & Media & $\mathrm{N}$ & $\begin{array}{c}\text { Desv. } \\
\text { Desviación }\end{array}$ & $\begin{array}{c}\text { Desv. } \\
\text { Error } \\
\text { promedio }\end{array}$ \\
\hline Par 1 & DC1 & 6.0278 & 152 & .99045 & .08034 \\
& DA2 & 5.9961 & 152 & .97477 & .07906 \\
Par 2 & DA2 & 5.9961 & 152 & .97477 & .07906 \\
& DB1 & 5.8454 & 152 & 1.27343 & .10329 \\
Par 3 & DB1 & 5.8454 & 152 & 1.27343 & .10329 \\
& DD1 & 5.8048 & 152 & 1.01618 & .08242 \\
Par 4 & DD1 & 5.8048 & 152 & 1.01618 & .08242 \\
& DA1 & 5.6671 & 152 & 1.07788 & .08743 \\
\hline
\end{tabular}

Fuente: Elaboración propia basada en los datos obtenidos del estudio.

Se obtienen los valores de t para cada par de muestra, así como el intervalo de confianza de las diferencias para cada muestra, del mismo modo se muestran los valores $p$ menores a .05. Los resultados para cada par de muestras se presentan en la Tabla 3.

TABLA 3.

Prueba de muestras emparejadas para docentes.

\begin{tabular}{|c|c|c|c|c|c|c|c|c|}
\hline & \multicolumn{5}{|c|}{ Diferencias emparejadas } & $\mathrm{t}$ & $\mathrm{gl}$ & Sig. \\
\hline & \multirow[t]{2}{*}{ Media } & \multirow[t]{2}{*}{ Desv. } & \multirow[t]{2}{*}{$\begin{array}{l}\text { Desv. } \\
\text { Error } \\
\text { prom. }\end{array}$} & \multicolumn{2}{|c|}{$\begin{array}{l}95 \% \text { de int. de } \\
\text { conf. de la dif. }\end{array}$} & & & \\
\hline & & & & Inf. & Sup. & & & \\
\hline DC1-DA2 & .03173 & .73460 & .05958 & -.08600 & .14945 & .532 & 151 & .595 \\
\hline DA2-DB1 & .15066 & 1.11709 & .09061 & -.02836 & .32968 & 1.663 & 151 & .098 \\
\hline DB1-DD1 & .04057 & 1.05625 & .08567 & -.12870 & .20984 & .474 & 151 & .637 \\
\hline DD1-DA1 & .13772 & .78863 & .06397 & .01133 & .26410 & 2.153 & 151 & .033 \\
\hline
\end{tabular}

Fuente: Elaboración propia basada en los datos obtenidos del estudio.

Con la finalidad de obtener la relación de competencias que fueron valoradas como más importantes por los docentes se ordenaron de mayor a menor organizadas por apartado considerando el mayor promedio de evaluación. Se presenta en la Tabla 4 la lista de competencias que se ubicaban por arriba de la media de cada factor encontrando. 
TABLA 4.

Competencias por arriba de la media para la muestra de docentes.

\begin{tabular}{|c|c|}
\hline & Media \\
\hline $\begin{array}{l}\text { C3.-Capacidad para participar en clúster, redes empresariales, o colaborar con universidades para } \\
\text { desarrollar el aprendizaje tecnológico }\end{array}$ & 6.16 \\
\hline C4.-Desarrollar productos y servicios innovadores para mercados globales & 6.10 \\
\hline $\begin{array}{l}\text { C5.-Saber desarrollar contenidos digitales reutilizables en distintas plataformas y dispositivos } \\
\text { móviles }\end{array}$ & 6.04 \\
\hline $\begin{array}{l}\text { C1.-Definir la estrategia de innovación apoyada en las TIC asegurando las funcionalidades, la } \\
\text { mejora del servicio y de los procesos de negocio }\end{array}$ & 6.04 \\
\hline C2.-Capacidad de organizar la colaboración interno-externa para la innovación & 6.03 \\
\hline $\begin{array}{l}\text { C9.-Coordinar el contacto físico con el cliente con el uso de plataformas tecnológicas y redes } \\
\text { sociales }\end{array}$ & 6.02 \\
\hline $\begin{array}{l}\text { A1.-Comprender cómo las tecnologías digitales permiten la producción de conocimiento como } \\
\text { recurso económico }\end{array}$ & 6.41 \\
\hline $\begin{array}{l}\text { A2.-Saber utilizar la información como una mercancía que se puede comercializar en soportes } \\
\text { digitales }\end{array}$ & 6.06 \\
\hline $\begin{array}{l}\text { B7.-Conocer las tendencias tecnológicas de cada momento para mejorar la productividad o abrir } \\
\text { nuevas líneas de negocio }\end{array}$ & 6.21 \\
\hline B3.-Entender la información relevante sobre Internet para la toma de decisiones & 6.18 \\
\hline B2.-Implantar sistemas de vigilancia e innovación tecnológica para no perder la competitividad & 6.03 \\
\hline B1.-Conocer la arquitectura y usos de Internet necesaria para el funcionamiento del negocio & 5.96 \\
\hline B6.-Entender la información relevante sobre hardware y software para la toma de decisiones & 5.91 \\
\hline B4.-Conocer la arquitectura de hardware/software necesario para el funcionamiento del negocio & 5.78 \\
\hline B5.-Implantar sistemas de vigilancia e innovación tecnológica para no perder la competitividad & 5.73 \\
\hline D9.-Desarrollar relaciones con los empleados más basadas en la confianza que en la supervisión & 6.01 \\
\hline $\begin{array}{l}\text { D4.-Elegir el modelo adecuado de organización de la empresa para aumentar la productividad con } \\
\text { las TIC }\end{array}$ & 6.01 \\
\hline $\begin{array}{l}\text { D6.-Desarrollar organizaciones globales descentralizadas geográficamente y con grupos de trabajo } \\
\text { multidisciplinares }\end{array}$ & 5.92 \\
\hline $\begin{array}{l}\text { D7.-Organizar la producción y los servicios en flujos de trabajo en función de los clientes, socios y } \\
\text { proveedores }\end{array}$ & 5.89 \\
\hline $\begin{array}{l}\text { D3.-Gestionar organizaciones flexibles, dispersas geográficamente en las que el proceso de } \\
\text { producción se realiza de forma virtual }\end{array}$ & 5.82 \\
\hline $\begin{array}{l}\text { A15.-Gestión de la demanda: implantar soluciones de negocio automatizadas por medio de las TIC } \\
\text { para gestionar la demanda de servicios }\end{array}$ & 5.91 \\
\hline A14.-Saber valorar y gestionar los activos intangibles & 5.86 \\
\hline $\begin{array}{l}\text { A10.-Aumentar el contenido de información de los productos físicos para añadir más valor a los } \\
\text { mismos o crear nuevos productos y servicios }\end{array}$ & 5.76 \\
\hline $\begin{array}{l}\text { A13.-Conseguir fuentes de financiación para los proyectos más innovadores a través de capital } \\
\text { riesgo, bussiness angels, capital semilla y otros }\end{array}$ & 5.76 \\
\hline A12.-Gestionar de manera coordinada la cadena de valor física y la virtual & 5.73 \\
\hline A11.-Sustituir actividades físicas de la cadena de valor por actividades virtuales con menor costo & 5.67 \\
\hline
\end{tabular}

Fuente: Elaboración propia basada en los datos obtenidos del estudio. 
En la Tabla 5 se muestran los valores $F$ y significancia del análisis de ANOVA con el método de comparaciones múltiples de Tukey con un nivel de confiabilidad del 95\% para cada uno de los factores que se obtuvieron de las encuestas como la universidad donde labora, nivel máximo de estudios, categoría profesional, tamaño de la empresa y tipo de organización.

TABLA 5.

Valores de ANOVA entre grupos.

\begin{tabular}{ccccccccccc}
\hline & \multicolumn{2}{c}{$\begin{array}{c}\text { Universidad donde } \\
\text { labora }\end{array}$} & \multicolumn{2}{c}{$\begin{array}{c}\text { Nivel Max } \\
\text { Estudios }\end{array}$} & \multicolumn{2}{c}{$\begin{array}{c}\text { Categoría } \\
\text { Profesional }\end{array}$} & \multicolumn{2}{c}{$\begin{array}{c}\text { Tamaño } \\
\text { Empresa }\end{array}$} & \multicolumn{2}{c}{$\begin{array}{c}\text { Tipo de } \\
\text { Organización }\end{array}$} \\
\hline & F & Sig. & F & Sig. & F & Sig. & F & Sig. & F & Sig. \\
\hline DA1 & 1.892 & .115 & .527 & .756 & 1.941 & .091 & .165 & .975 & 1.056 & .370 \\
DA2 & 1.481 & .211 & .865 & .506 & 1.309 & .263 & .363 & .873 & 1.250 & .294 \\
DB1 & 1.833 & .126 & .237 & .946 & 1.886 & .100 & .591 & .707 & 1.436 & .235 \\
DC1 & 1.183 & .321 & 1.059 & .386 & 1.957 & .088 & .129 & .986 & 1.051 & .372 \\
DD1 & 1.578 & .183 & 1.612 & .160 & 2.132 & .065 & .422 & .833 & .712 & .546 \\
\hline
\end{tabular}

Fuente: Elaboración propia basada en los datos obtenidos del estudio.

La muestra de docentes con el método utilizado para todos los factores el valor $p$ fue mayor que el nivel de significancia, por lo que no se cuenta con suficiente evidencia para rechazar la hipótesis de que las medias de población son todas iguales.

\section{DiSCUSIÓN}

Los resultados de este estudio coinciden con las prospectivas que plantea el WEF (2016) ubicando con mayor importancia a las competencias relacionadas con la innovación, los nuevos productos y clientes sobre la economía global, el uso de las TIC, los cambios organizacionales y los modelos de negocios digitales. Sin embargo, no se presenta diferencias significativas en la importancia de cada una de estas competencias. Las diferencias estadísticamente significativas nos indican que existe una diferencia, pero no el tamaño de estas. Así mismo, el WEF (2016) se ha encargado de proporcionar la información necesaria a las comunidades académicas sobre las habilidades que requieren los trabajadores del futuro sin presentar un orden de importancia.

En el contexto mexicano, la SEECO (2016) manifiesta como fortalezas del desarrollo de la Industria 4.0 para el país la oferta educativa especializada, la economía de servicios, industria de alta tecnología y recursos humanos creativos. Los docentes concuerdan con altos niveles de importancia para todas las competencias relacionadas con estas fortalezas. Son las competencias relacionadas con la gestión, aplicación y financiación de nuevos modelos de negocios basados en la economía digital y los activos intangibles, las que se muestran con menor importancia para los docentes.

Autores como Carrizosa (2018) establecen la necesidad de brindar importancia a los entornos de aprendizaje diversos y disruptivos; y López (2019) quien indica que la importancia de la innovación en las universidades y de obtener soluciones a problemas reales, 
comparten el foco de interés con los resultados de este estudio en el cual los profesores señalan con mayor importancia las competencias relacionadas con la innovación, creación de nuevos productos y clientes; principalmente con la forma de vincular de manera disruptiva los procesos productivos con los educativos. Sin embargo, dejan como último lugar de importancia las competencias para conseguir fuentes de financiamiento para proyectos innovadores, la coordinación de la cadena de valor física y virtual, así como la sustitución de actividades físicas por virtuales de menor costo.

Las competencias relacionadas con la economía global, de acuerdo con las divisiones de la Industria 4.0, realizadas por Franco (2015), presentan la comprensión de individualización, descentralización y creación de redes que explica el autor. Estas competencias consideradas por los docentes como las segundas más importantes, al no existir evidencia de la diferencia de importancia con las otras competencias mantienen la incertidumbre del alcance de los cambios esperados en este modelo productivo.

La importancia que los profesores otorgan a las competencias relacionadas con las TIC respalda lo señalado por Hirsch (1996), en cuanto a que lo técnico es un elemento fundamental para el logro de la globalización; y lo mencionado por Herrero (2014), quien indica una relación directa entre la utilización de éstas por parte de los docentes con los alumnos.

Por otra parte, las visiones del Foro Económico Mundial (WEF, 2016), López y Escudero (2016) y Pineda (2020) van dirigidas hacia que los cambios organizacionales y la naturaleza del trabajo serán los que caractericen a esta revolución industrial; en este estudio los resultados arrojan que las competencias relacionadas con la organización de las empresas digitales y las TIC no tienen diferencias significativas. Promover las competencias relacionadas con estos cambios organizacionales y modelos de negocio debe incluirse en la labor docente.

Las competencias digitales directivas adquiridas durante la formación profesional son las que serán herramientas básicas para la transformación digital de las empresas. Según Carruyo et al. (2017), la participación de las universidades es fundamental en este proceso de integración tecnológica. La visión de los docentes de las áreas de ingeniería y negocios se observa sin diferencias significativas, por lo que se puede asumir una visión unificada hacia los cambios futuros.

En la industria 4.0 la participación de los centros de generación y aplicación del conocimiento y de las IES ha logrado un papel fundamental en la formación de los profesionales, como mencionan Cobo (2010), Quintero (2017) y López (2019). Con base en estos autores, la participación de las IES se verá en la cúspide de su desarrollo en el momento que se involucren de manera colaborativa en la mejora de la producción de bienes y servicios con las industrias. Los docentes que participaron en este estudio ubican a esta capacidad para participar en clúster, redes empresariales o colaborar entre universidades en el desarrollo del aprendizaje tecnológico como la competencia con la mayor media de nivel de importancia.

Priorizar las competencias digitales directivas como se presenta por los docentes, permite generar estrategias que brinden una formación de talentos que fortalezcan los procesos de una transformación digital en términos tecnológicos. Sin embargo, al evaluar con menor valor de importancia las competencias relacionadas con la gestión, aplicación 
y financiación de nuevos modelos de negocio basados en la economía digital y los activos intangibles, se corre el riesgo de ausencia de talentos para la generación y desarrollo de nuevas empresas digitales. En ese sentido, de acuerdo con la visión de los docentes los talentos formados se enfrentarán al reto de ser empleados altamente capacitados en vez de dirigentes empresariales.

\section{CONCLUSIONES}

Los cambios laborales que se enfrentarán en un futuro cercano presentan retos importantes para los profesionales que se encuentran en formación en el presente. La participación de los docentes es primordial en la formación de competencias que requerirán las empresas en el proceso de cambio.

El valor de las medias del nivel de importancia que otorgan los docentes a estas competencias está dirigidas en primer lugar a la innovación, los nuevos productos y los clientes; principalmente en el proceso de colaboración e intercambio de conocimiento entre pares y centros productivos, la vinculación de teoría y la práctica en un mundo digital y globalizado. Le siguen las competencias relacionadas con las tendencias en TIC y el uso adecuado del Internet, el conocimiento del funcionamiento de las redes digitales y las nuevas tecnologías.

Se muestra un nivel de importancia menor para la organización de las empresas digitales, la comunicación y las formas flexibles de trabajo entre el personal. Estos niveles de importancia indican la atención proporcionada a los temas relacionados con la Industria 4.0 para el proceso de enseñanza-aprendizaje. Sin embargo, no existe evidencia para declararlas con diferencias significativas.

De esta forma, para las competencias mejor evaluadas se propone diseñar estrategias académicas adecuadas para fomentar la vinculación efectiva con las empresas para su conocimiento; en el mismo sentido para las de menor valor de importancia, diseñar acervos informativos relacionados a estas competencias para mejorar su percepción.

El alcance de los beneficios de la Industria 4.0, se presenta en el balance entre tecnología, conocimiento y disrupción de modelos de negocios. Las competencias relacionadas con la gestión de nuevos modelos de negocio y activos intangibles de las empresas digitales, sí presentan diferencias significativas con respecto a la que se encuentra en las competencias relacionadas con la organización de empresas digitales, siendo la de menor nivel de importancia. Para poder alcanzar el desarrollo de proyectos innovadores se requerirán estas competencias para poder financiarlos y agregar valor a través de los activos intangibles de universidades y empresas.

Los docentes no presentan diferencias significativas en el análisis de varianzas, lo que demuestra la homologación de los profesores con el proceso de adopción del fenómeno digital empresarial desde la perspectiva académica de las áreas de ingeniería y negocios, las universidades donde laboran, los niveles de estudio y el tipo de organización en la participan. Aprovechar esto se debe considerar para la generación de políticas públicas que impulsen el desarrollo regional a través de la industria 4.0. 


\section{REFERENCIAS}

Area, M. (2010). ¿Por qué formar en competencias informacionales y digitales en la educación superior? RUSC. Universities and Knowledge Society Journal, 7(2), 2-5. http:// dx.doi.org/10.7238/rusc.v7i2.976

Bauman, Z. (2003). Modernidad Líquida. México, D.F.: Fondo de Cultura Económica.

Bernal, C. (2010). Metodología de la investigación. Bogotá, D.C.: Pearson educación.

Carrizosa, E. (2018). Lifelong Learning e Industria 4.0. Elementos y requisitos para optimizar el aprendizaje en red. Revista Internacional y Comparada de Relaciones laborales y derecho del empleo, 6(1), 40-63. Disponible en http://ejcls.adapt.it/index.php/ rlde_adapt/article/view/550

Carruyo, N., Ureña, Y. C. y Quiñones, E. (2017). Empoderamiento digital: Integración Universidad, Empresa y Políticas públicas para el desarrollo endógeno. Scientific ejournal of Management Science, 12(36), 20-34. https://doi.org/10.5281/zenodo.4903922

Castells, M. (2010). Globalización e identidad. En, S. Florensa (Ed), Quaderns de la Mediterrània No. 14 Diez años de diálogo mediterráneo (pp. 11-20). Barcelona: IEMed.

Castillo, A. (2016). Industria 4.0 Rol de las redes sociales para conectar a las empresas en torno a una economia circular. [Tesis de maestría]. Universidad de Cantabria, Cantabria, España. Disponible en http://hdl.handle.net/10902/8655

Cobo, C. (2010). Cultura digital y nuevos perfiles profesionales: desafíos regionales. @tic, (5), 1-7. https://doi.org/10.7203/ATTIC.5.187

Estados Unidos Mexicanos. Secretaria de Economía. (2016). Crafting the Future: a roadmap for industry 4.0 in México. México, D.F.: SEECO/Prosoft 3.0. Disponible en https://amiti.org.mx/5404/crafting-the-future-a-roadmap-for-industry-4-0-in-mexico

Franco, D. (2015). Utilización del Modelo de Triple Hélice para el desarrollo de nuevos sectores productivos en el contexto de la Industria 4.0. [Tesis de maestría]. Universidad del País Vasco, Pais Vasco, España. Disponible en http://hdl.handle.net/10810/15735

Herrera, R. e Hidalgo, A. (2019). Dinámica de la gestión de la innovación de servicios y co-creación en empresas del sector economía digital. Contaduría y Administración, 64(1), 1-20. http://dx.doi.org/10.22201/fca.24488410e.2018.1802

Herrero, R. (2014). El papel de las TIC en el aula universitaria para la formación en competencias del alumnado. Pixel-Bit. Revista de Medios y Educación, (45), 173-188. Disponible en https://recyt.fecyt.es/index.php/pixel/article/view/61639

Hirsch, J. (1996). ¿Qué es la globalización? En, G. Ávalos (Ed.), Globalización, capital y Estado (pp. 83-93). México, D.F.: UAM-X.

IBM. (versión 27.0). Statistical Package for Social Sciences (SPSS). [Software]. Armonk: IBM. Disponible en https://www.ibm.com/mx-es/analytics/spss-statistics-software

INEGI. (2017). Encuesta Nacional sobre Disponibilidad y Uso de Tecnologías de la Información en los Hogares (ENDUTIH 2017). [Online]. Disponible en https://www. inegi.org.mx/programas/dutih/2017/ 
ITU. (2018). Conjunto de herramientas para las habilidades digitales. Suiza: ITU.

Lombardero, J. L. (2015). Problemas y retos de gestión empresarial en la economía digital: estudio comparado y sistémico de competencias directivas. [Tesis doctoral]. Universidad Camilo José Cela, Madrid, España. Disponible en http://hdl.handle. net/20.500.12020/181

López, A. (2019). Transformación digital e innovación global en la Universidad 4.0. Economistas, (165), 113-122. Recuperado de https://www.cemad.es/wp-content/ uploads/2019/10/Transformacion-digital-innovacion-universidad-4-0.pdf

López, J. y Escudero, V. (2016). Industria 4.0, la gran oportunidad. Economía Aragonesa, (59), 109-123.

Naji, M. J. (2018). Industria 4.0, competencia digital y el nuevo Sistema de Formación Profesional para el empleo. Revista Internacional y Comparada de Relaciones Laborales y Derecho del Empleo, 6(1), 164-194. Disponible en http://ejcls.adapt.it/index. php/rlde_adapt/article/view/555

Pineda, A. (2020). La comunicación organizacional en la gestión empresarial: retos y oportunidades en el escenario digital. Revista GEON (Gestión, Organizaciones y Negocios), 7(1), 9-25. https://doi.org/10.22579/23463910.182

Quintero, M. G. (2017). Las nuevas necesidades formativas en el marco de la digitalización y demás avatares 4.0. (La agenda 2030 y los objetivos de desarrollo sostenible como herramientas docentes). Lan Harremanak Especial - Revista de relaciones laborales, (37), 128-149. http://dx.doi.org/10.1387/lan-harremanak.18409

Velásquez, A. (2020). Prospectiva geopolítica sobre civilizaciones: el nuevo orden mundial se reconfigura. En, J. Ángel y D. Pérez (Coords.), Globalización, constitucionalismo y cultura de paz (pp. 29-41). México, D.F.: UniLibre/UABC.

Vera-Noriega, J., Rodríguez-Carvajal, C. y Martínez-García, E. (2017). Capital Cultural y competencias digitales en estudios universitarios. Ventana Informática, (36), 99-116. https://doi.org/10.30554/ventanainform.36.2389.2017

Weller, J. (2020). La pandemia del COVID-19 y su efecto en las tendencias de los mercados laborales. [LC/TS.2020/67]. Santiago: CEPAL. Disponible en https://www.cepal. org/es/publicaciones/45759-la-pandemia-covid-19-su-efecto-tendencias-mercados-laborales

WEF. (2016). The Future of Jobs: Employment, Skills and workforce strategy for the fourth Industrial Revolution. Switzerland: WEF. Available from https://www3.weforum.org/ docs/WEF_Future_of_Jobs.pdf

Zempoalteca, B., Barragán, J., González, J. y Guzmán, T. (2017). Formación en TIC y competencia digital en la docencia en instituciones públicas de educación superior. Apertura Universidad de Guadalajara, 9(1), 80-96. http://dx.doi.org/10.32870/ Ap.v9n1.922 
Jorge Carlos Canto Esquivel es Ingeniero en Electrónica. Maestro en Ciencias en Planificación de Empresas y Desarrollo Regional. Doctor en Ciencias Sociales. ProfesorInvestigador de tiempo completo enfocado a la innovación tecnológica. ORCID: https://orcid. org/0000-0002-5599-8870

Jennifer Mul Encalada es Doctora en Ciencias de la Administración por la Universidad Nacional Autónoma de México (México). Cuenta con el perfil Prodep y es socia de la Academia de Ciencias Administrativas-AC y del Colegio de Posgraduados en Ciencias Administrativas, AC. Su línea de investigación es "Organización y Sociedad", en la cual ha realizado estudios sobre gestión del conocimiento e innovación, responsabilidad social empresarial, competitividad de las pymes y empresas familiares, a partir de los cuales ha publicado diversos capítulos de libros, artículos y ponencias. ORCID: https://orcid.org/00000003-0034-8165

Ruth Noemí Ojeda López es Doctora en Ciencias de la Administración p o r l a Universidad Nacional Autónoma de México (México). Profesora de la Universidad Autónoma de Yucatán (México), siendo su línea de investigación "sociedad y organización” de la cual han derivado libros, capítulos de libro, artículos y memorias en extenso. Por sus actividades docentes cuenta con la certificación de la ANFECA así como el perfil Prodep, en el ámbito de la investigación es miembro del Sistema Nacional de Investigadores nivel 1. ORCID: https://orcid.org/0000-0002-7137-120X 